\title{
The Clinical Application of Body Language Communication in Pediatric Nursing Work
}

\author{
Yaping Shi ${ }^{1}$ \\ ${ }^{1}$ Taihe Hospital, Shiyan, Hubei Province, China
}

Keywords: body language; communication; children; nursing work

\begin{abstract}
To explore the clinical application of body language communication in pediatric care, a total of 160 children, aged 1 to 10 years, were randomly divided into observation group and control group, 80 cases each. The control group used the conventional nursing model. The observation group strengthened body language communication on the basis of the control group. The control group had the condition of compliance with the doctor's care before and after nursing, and the satisfaction rating of nursing care. Results show that body language communication can not only strengthen the children's willingness to comply with doctor's orders, but also improve the satisfaction of nursing. Therefore, body language communication has important clinical significance in pediatric nursing work.
\end{abstract}

\section{Introduction}

With the development of modern medicine, pediatric nursing can not only use language communication but also non-verbal communication. Non-language communication skills include: nurses' instruments, listening skills, body language, body language communication, etc. [1]. In the process of pediatric care, children must not only face the children who cannot correctly describe the symptoms and needs of the disease, but also need to face the anxiety, irritability, naughtiness, and high demands of their parents[2]. In this regard, in the process of child care, you need to have the right communication skills.

Body language is also called posture. It refers to the body language that expresses emotions and conveys information through the usage of body postures and movements in specific situations. Although it is a non-linguistic factor, it contains follow-up, visibility, and authenticity. It can be used in practical applications. Make up for the shortcomings of the language "incomplete" in the transmission of information. Studies have found that smiling faces in nursing can relieve the tension of patients and increase trust in nurses, virtually narrowing the gap between nurses and patients [1]. The application of body language communication in pediatric nursing can increase the compliance of the children's doctor's advice and improve the satisfaction of nursing work, which is worthy of clinical application.

\section{Body Language Communication and Pediatric Nursing}

Language communication always runs through the entire nursing work. Nurses should be good at guiding patients to speak. The key is to see if the nurse has compassion for the child. At the same time, the nurses should be keen to observe the power of their children, and be comfortable, playful, lively, emotional, and straightforward. To become their good friends, encourage more language and do not scold children for protection. Before the nursing operation, you can also use the stimulating method and the contrast method, tell them the stories of the same animals, use the bravery of the imaginary animals in exchange for their bravery, so as to achieve the purpose of communication [2].

Communication Skills Necessity for Pediatric Nursing Pediatrics is an independent department of the hospital and has special characteristics that differ from those of other departments. Children are the future of the motherland, and they are also the young emperors at home. They express their emotions straightforwardly and simply. When they are ill and hospitalized, they have uncooperative behaviors in nursing technology operations. The nurse must cooperate with doctors in treating 
children's body diseases and taking into account the psychological needs of children. It also needs to take into account the emotional needs of multiple families. It should provide appropriate care according to the psychological characteristics of children at different ages and the different psychological responses of children with diseases. As shown in Figure 1. Pediatric nursing work is more complicated than adult care. In terms of pediatrics, with the improvement of people's cultural living standards and the change of health concepts, parents' awareness of children's protection has been constantly enhanced, and the demands for nursing work are also increasing. Therefore, the occurrence of nursing disputes is In an upswing, in order to eradicate nursing disputes in the bud, in clinical nursing work, we found that many of the causes of disputes are directly or indirectly related to inadequate communication between nurses and patients, and disputes that really belong to nursing errors or care accidents are Minimal, for this reason, how to prevent the occurrence of nursing disputes in advance, it is extremely important to make good communication between nurses and patients.

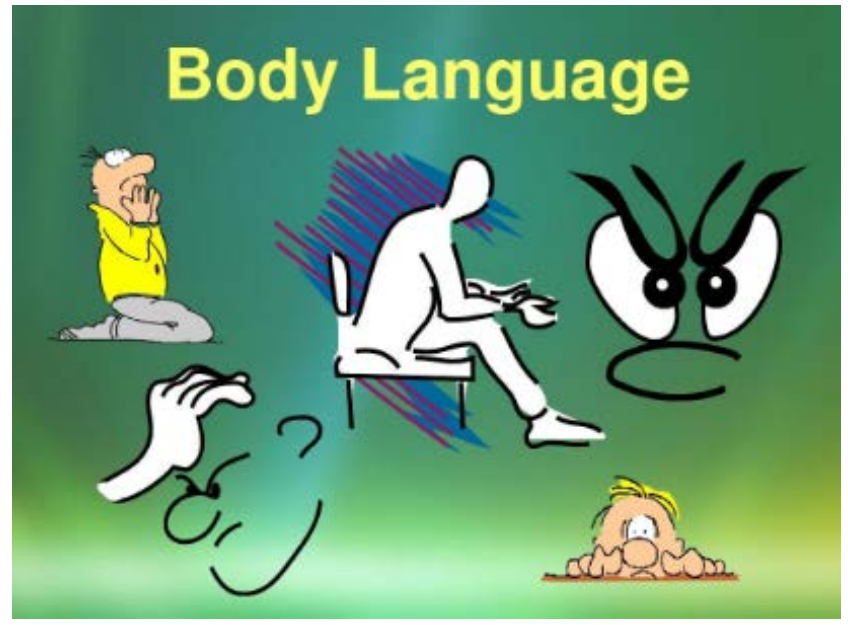

Fig.1 body language

\section{Body Language Communication}

1) Language-based communication is the use of language expressions. It is expressed in the form of a smile, a cry or a shout to achieve the purpose of communication. After 6 years of age, children can gradually use a language with a more complete thinking, which is suitable for language-based communication.

2) Non-verbal communication. Non-verbal communication is also called body language communication. It refers to the use of gestures, movements, facial expressions, posture changes, and reactions to communicate with each other. Before the age of 6 , the children's language skills were poor, and it was difficult to communicate with them. In addition, intellectual development had not yet reached a full understanding of abstract things. Therefore, most of the communication with the outside world was in non-verbal communication.

3) Abstract communication. Abstract-style communication expresses communication in the form of game signs, photos, appreciation ability, clothing selection, and painting. This type of communication is suitable for age spans and is more suitable for older children.

4) The opening quiz is not available in closed quizzes. With open quizzes, more information can be obtained, which facilitates further communication. If you can ask: What is your reaction to taking medicine? Similar are: What is ? / How is it?

5) In order to win the cooperation of children, avoid using such nonverbal words as no/no/no, positive contact has the best effect. For example, when children are found eating crayons, they should be explained: Crayons are used for drawing. And don't say: / Don't eat crayons, etc.

6) When directing children to correctly choose abstract things, they can use positive direct contact. For example, let the children take off their coats and do a chest auscultation. Avoid saying: Would you like to take off your coat? This kind of questioning will make children feel that they cannot take 
off their coats. Instead, you should say: / Take off your coat to auscultator your chest, Do you want to take it off?

7) For young children, do not think that they cannot understand the language of adults. When they do not cooperate with care, it is difficult to predict that some blunt, especially emotional speeches, bad discussions and expressions will have an impact on young children. .

8) When the children's family members are present and express disagreement or dissatisfaction with the process of care and communication, it is best to avoid the children's communication with their loved ones and then communicate with the children so as to avoid any confusion.

9) Encourage children with relatives to join the game. In the game, contact with the children and nursing staff. At the same time assess the safety of the toy and determine the amount of activity based on the condition and the environment.

10) Use the theory of child development rather than the theory of healthy children to judge, interpret, and analyze their behavior.

11) Encourage children to correctly express fear, anxiety, general feelings and problems. It also allows children to be immature due to stress.

12) Discussion With the development of modern medical science, the nursing model has been changed from/centered on disease/patient-centered/holistic care. Good communication with children is an important part of the pediatric overall nursing model in modern medical models. , is one of the important topics that pediatric care workers pay attention to and explore [1].

As a child suffering from diseases, in addition to regular medical care, in the course of diagnosis and treatment, pediatric nurses should give them social and psychological care and avoid violent compulsory care, so that they can not only enable the children to coordinate and cooperate with the treatment. Therefore, to complete this kind of biological 2 physiological 2 social medical model of medical care, it is extremely important to have good communication with the children. It is worth mentioning that the majority of pediatric care workers are aware that games play an important role in communication. The game can achieve the following purposes: to help children recognize the feelings of themselves and the communicator; to make the children get a new sense of communication; so that children can turn their attention to the game to reduce the anxiety of the communicators brought about by the nursing operation; Can help the caregiver to successfully inform the children of all kinds of injurious treatment process; Through the game can help the caregiver to assess the character of children, using appropriate means of communication.

\section{The Effect of Body Language Communication in Pediatric Nursing Work}

Because young patients are unfamiliar with the diagnostic procedures and fears of drugs and intravenous injections, they often have conflicting emotions in the course of clinical diagnosis and treatment. This type of emotion affects their compatibility, resulting in a more severe course of disease treatment. Severe crying and struggling are not only unfavorable for diagnostic accuracy, but may also lead to medical accidents during the injection process [5]. Body language communication is an emerging model in nursing operations in recent years. Because children are young and simple language communication may not work well, body language has become an important means of communication.

Based on this background, this article uses a comparative approach to study the role of body language communication in improving the compliance of children, and now makes the following report:

\subsection{General examples.}

The 160 children with pediatric treatment in a hospital from May 2016 to November 2016 were randomly divided into two groups. In the control group, there were 80 males, 40 males and 40 females,

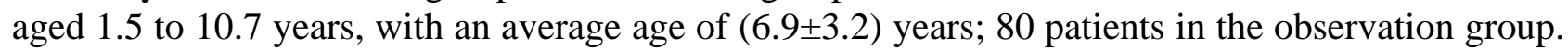
There were 40 males and 40 females, aged from 1.6 to 10.0 years old, with an average of (6.6 \pm 3.6$)$ years of age. There was no statistically significant difference between the two groups in terms of sex ratio, average age, and disease type distribution. $>0.05$ ). 


\subsection{Methods.}

The control group adopted regular language communication mode; the observation group was supplemented by language communication mode on the basis of language communication. The contents of body language communication include:

1) Smile with care [3], so as to create a good communication atmosphere before the nursing work is implemented. However, when the child is critically ill or the parents are in a hurry, if the nursing staff is still smiling, it will cause the children's family members to resent, or even cause unnecessary disputes. Therefore, during the nursing process, the nursing staff should use facial expressions properly and combine them. Changes in the heart of children and their families are timely adjustment of facial expressions.

2) To take care of every child so that children and their families recognize that they are valued, and use body language to create an equal and peaceful environment [4], and maintain a beautiful posture during the conversation with the families of children.

3) Behave meticulously, such as touching the forehead of a child in a nursing job, checking for fever, and letting children feel caring.

4) Incentive support is given in due course. For example, if a child with lumbar puncture wear is stressed and frustrated because of fear of pain, the nurse can hold the child's little hand, which is undoubtedly a silent stimulus.

5) Neat clothing and refreshed spirits form a beautiful image of white angels, which can bring them closer to their children. The hands and feet are gentle and the movements are familiar during the nursing operation, which enhances the trust and security of parents and children.

6) Good at using eyes and eye contact. Encouragement, appreciation of the eyes and other information transmission can effectively enhance the effectiveness of communication. The first thing the child can come in contact with is the nurse's eyes. Sincere and sincere eyes on the child help enhance the sense of closeness with the child and the parent, which is conducive to the smooth development of the nursing work.

\subsection{Evaluation criteria.}

The children's compliance with the doctor's compliance and acceptance behavior scores were assessed using the grading standards reported by Sun Qinzhou [5] et al.

1) Completely contradictory (0 points), showing rejection of treatment, crying, fear, and non-cooperation.

2) Partial conflict (2 points), willing to receive treatment but not cooperate, manifested as partial resistance but not escape.

3) Partially accepted (6 points), healed but partial cautious, willing to follow the doctor's advice but sometimes conservative.

4) Absolutely accepted (10 points), doctor-patient relationship harmonious, and not afraid of the treatment process and environment [ 3], the quality of nursing scores using the nursing care quality rating scale developed by the hospital management department and nursing department, out of 100 points, referring to Yan Lin et al. [4] designing family members and children's nursing satisfaction survey questionnaire.

\subsection{Statistical methods.}

Measurement data using t test, count data using x2 test, $\mathrm{p}<0.05$ for the difference was statistically significant.

\section{Results and Discussion}

The comparison of the compliance of doctors in the two groups showed that there was no significant difference between the two groups in partial conflict rate and partial acceptance rate ( $>0.05$ ), but the complete conflict rate in the observation group was $5.6 \%$, which was significantly lower than that in the control group. The total acceptance rate was $45.6 \%$, which was significantly 
higher than that of the control group (35\%). The difference was statistically significant $(\mathrm{p}<0.05)$. See Table 1. The nursing quality score of the observation group was (93.7 \pm 5.3$)$ points, which was significantly higher than that of the control group $(83.4 \pm 4.7)$ points $(p<0.05)$.

Table 1 Comparison between doctors and children in two groups (\%)

\begin{tabular}{|c|c|c|c|c|}
\hline Groups & Fully accepted & $\begin{array}{c}\text { Partially } \\
\text { accepted }\end{array}$ & Partial conflict & $\begin{array}{c}\text { Completely } \\
\text { contradict }\end{array}$ \\
\hline $\begin{array}{c}\text { Control } \\
\text { group(n=80) }\end{array}$ & $23(28.75)$ & $27(33.75)$ & $18(22.50)$ & $12(15.00)$ \\
\hline $\begin{array}{c}\text { Observation } \\
\text { group }(n=80)\end{array}$ & $30(37.50)$ & $26(32.50)$ & $14(17.5)$ & $10(12.5)$ \\
\hline
\end{tabular}

Compared with the control group, $\mathrm{p}<0.05$

The special nature of pediatric nursing subjects complicates the nursing work. In order to establish a harmonious nurse-patient relationship, new nursing models should be continuously explored in the clinic [5]. The study found that, in some cases, language-only communication can hardly meet the expectations of children and their families and reduce the compliance of doctors.

Body language is more suitable for younger patients than simple language. Because children are naturally agitated and have fears in hospitals, nursing operations are relatively difficult. The focus should be on helping children with diagnosis and treatment. , to reduce the level of irritability or fear is the key to care [4].

The results of this study proved that after the body language communication, the children's fear of treatment can be effectively reduced, and thus more in line with the clinician's examination and treatment. In this study, the use of touching the head and other movements can help the child to comfort and comfort. Refueling gestures can encourage the children to be afraid of treatment, and the resistance can be effectively reduced. At the same time, the facial expressions of the nursing staff also affect the emotions of the children when they visit the doctor. Especially in the case of smiles, if the nursing staff keeps smiling and communicates with the children in soft language, it is more capable than serious language and facial expressions. The children were unloaded and the doctors were diagnosed and treated. The results of this study show that more children in the observation group using body language communication intervention care no longer have conflicting treatment emotions, and the compliance with diagnostic treatment is high. Only 14 cases have partial conflict, which proves that this kind of nursing intervention. The study also found that the application of body language communication has virtually improved the quality of care. The scores of the quality of care in the observation group were significantly higher than those in the control group. The improvement in the quality of care also increased the degree of satisfaction of the children and their families in nursing work.

\section{References}

[1] Q.Zh. Sun and J.B. Yan, Effects of body language communication on oral diagnosis and treatment of mentally handicapped children, Guangdong Dental Prevention and Treatment, 2005, vol.4, pp.80-81.

[2] W.Ch. Liang, Common causes and countermeasures of pediatric nurse-patient communication disorders, China Medical Frontier, 2009, vol.2, pp.115-116.

[3] L.T. Yan and Zh.H. Liu, Nurse-patient communication skills in pediatric patients, Chinese medical guide, 2010, vol.35, pp.151-152.

[4] P.T. Xie, Pediatric patient care problems and countermeasures, China Medical Herald, 2010, vol.7, pp.85-86.

[5] L.X. Song and B.T. Wang, Pediatric nurses and patients to communicate effect, Jilin Medicine. 2010, vol.11, pp.29-30.

[6] M.H. Li and Sh.Q. Zhang, Application of Body Language Communication, Nursing Practice and Research, 2008, vol.5, pp.32-33. 